Bol. Mus. Para. Emilio Goeldi, Ciências Naturais, Belém, v. 1, n. 3, p. 43-52, set-dez. 2006

\title{
Estrutura dos bosques de mangue da península de Bragança, Pará, Brasil ${ }^{1}$
} Structure of mangrove stands on the Bragança peninsula, Pará, Brazil

\author{
Adriana dos Santos Matni ${ }^{\mathrm{I}}$ \\ Moirah Paula Machado de Menezes ${ }^{\mathrm{I}}$ \\ Ulf Mehlig II
}

Resumo: O presente estudo visa a descrever da estrutura de três bosques de mangue (Acarajó, Furo do Meio e Furo Grande) na península de Bragança, nordeste do estado do Pará. Neste estudo foi utilizado o método do quadrante centrado (PCQM). Foi constatada a presença de três espécies típicas de mangue: Rhizophora mangle, Avicennia germinans e Laguncularia racemosa. Todos os bosques apresentaram valor de importância mais elevado para $R$. mangle. Na área Acarajó, há presença de indivíduos de diferentes portes, o que pode ser indício de um processo constante de renovação. Na área Furo do Meio, há vários indivíduos de porte médio, o que pode ser indício de uma renovação pretérita. Furo Grande trata-se de um bosque ainda em fase de crescimento, pois há árvores de porte médio e poucas de grande porte, como nas outras áreas. A regressão entre altura e diâmetro mostra que a partir de $30 \mathrm{~cm}$ de DAP o crescimento em altura cessa. A densidade de árvores dos bosques parece baixa quando comparada aos outros trabalhos desenvolvidos na região, porém, quando a distribuição das árvores nas classes de diâmetro e altura são observadas, percebe-se que os bosques apresentam árvores de grande porte. A análise de agregação mostra a existência de dois grupos de bosque, um formado por Furo do Meio e outro por Acarajó e Furo Grande

Palavras-chave: Manguezal. Estrutura de bosques. Bragança.

Abstract: The study describes the forest structure of three mangrove stands (Acarajó, Furo do Meio and Furo Grande) on Bragança peninsula, north-eastern Pará state, Brazil, using the point centered quarter method (PCQM). Three typical mangrove tree species were found: Rhizophora mangle, Avicennia germinans and Laguncularia racemosa. At all stands, $R$. mangle reached the highest importance values. At AC, we found individuals of various size classes, indicating possibly a constant rejuvenation process. At Furo do Meio, there were various individuals of medium size, indicating a peak of renovation event in the past. Furo Grande was characterised by a stand still in development, with mainly medium-sized trees and few larger specimens. The relationship between dbh and height showed that height growth is size limited. Tree density appeared low in comparison to other reports; however, examination of the dbh size class distribution reveals that the stands of the present investigation are mainly composed of comparatively large trees. Cluster analysis separated two groups, segregating Furo do Meio from both other sites (Acarajó, Furo Grande).

Keywords: Mangrove. Forest structure. Bragança.

I Universidade Federal do Pará. Campus de Bragança. Instituto de Estudos Costeiros. Laboratório de Biologia Vegetal. Bragança, Pará, Brasil(amantnii@yahoo.com.br)(moirah@ufpa.br).

II Zentrum für Marine Tropenökologie (Centro de Ecologia Marinha Tropical) (ulf.mehlig@gmx.net).

1 Publicado inicialmente em forma de relatório pelo Projeto MADAM n 98.

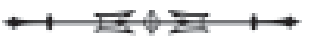




\section{INTRODUÇÃO}

Os manguezais de macromarés da costa nordeste do Pará e noroeste do Maranhão estendem-se da baía de Marajó, Pará até a Ponta de Tubarão, baía de São José, Maranhão, totalizando $650 \mathrm{~km}$ de litoral em linha reta (LACERDA, 1999), ocupando, somente no estado do Pará, 2177 km² (SOUZA FILHO, 2005). Estes manguezais desenvolvem-se atrás de praias e dunas, às margens das baías, em consórcio com restingas, nos estuários. Apesar da baixa diversidade de espécies arbóreas, os manguezais caracterizam-se por uma grande variabilidade espacial, como resposta às diferentes condições locais de salinidade, inundação e dinâmica costeira (PROST; RABELO, 1996). A península de Bragança, uma costa com diversas baías e estuários, localizada no nordeste do estado do Pará, apresenta cerca de $180 \mathrm{~km}^{2}$, dos quais $160 \mathrm{~km}^{2}$ constituem bosques de manguezal (KRAUSE; SCHORIES; DIELE, 2001). Estes bosques constituem um bom exemplo desta variabilidade fito-espacial, pois apresentam manguezais sob diferentes condições, desde alta salinidade e baixa inundação até manguezais de estuário sob a influência da água doce do rio Caeté. Portanto, este trabalho visou à descrição da estrutura e à identificação de possíveis diferenças estruturais em manguezais sob diferentes condições de salinidade e de inundação ao longo da península de Bragança, Pará, Brasil.

\section{MATERIAL E MÉTODOS}

\section{Área de estudo}

O estudo foi desenvolvido em três áreas de manguezais localizadas na península de Bragança, no estuário do rio Caeté, Bragança, Pará (Figura 1). A planície costeira bragantina apresenta cerca de 40 $\mathrm{km}$ de linha de costa, estendendo-se desde a ponta do Maiaú até a foz do rio Caeté (SOUZA FILHO, 1995). $\bigcirc$ acesso ao manguezal é facilitado pela rodovia PA-458, que corta cerca de $25 \mathrm{~km}$ de manguezal (CARVALHO, 2000). Nesta península foram escolhidas três áreas sob diferentes condições de salinidade e inundação:

Área 1 - Furo Grande (FG): localizada na porção norte da península, inundada aproximadamente 175 dias por ano e com salinidade intersticial entre $34 \mathrm{e}$ 42 (MENEZES; BERGER; WORBES, 2003).

Área 2 - Furo do Meio (FdM): localizada na porção central da península, inundada cerca de 120 dias por ano e com salinidade intersticial entre 17 e 42 (MENEZES; BERGER; WORBES., 2003).

Área 3 - Acarajó (AC): localizada a 6 km da cidade de Bragança à margem do rio Caeté, na comunidade do Acarajó, inundada cerca de 140 dias por ano e com salinidade intersticial entre 3 e 22 (MENEZES; BERGER; WORBES, 2003).

\section{Estrutura do bosque}

Para o estudo dos parâmetros estruturais foi utilizado o método do quadrante centrado (PCQM), segundo Schaeffer-Novelli e Cintrón (1986). A altura das árvores foi estimada, usando-se como base de referência uma régua de aproximadamente $6 \mathrm{~m}$ de altura. $\bigcirc$ diâmetro do fuste foi medido à altura do peito $(1,3 \mathrm{~m})$, sendo que em indivíduos de Rhizophora este parâmetro foi medido acima da última raiz escora. Árvores com diâmetro menor que $2,5 \mathrm{~cm}$ não entraram no inventário e aquelas com mais de um tronco foram consideradas como um único indivíduo. Com os dados de altura, diâmetro e distância entre os indivíduos, foram calculados parâmetros de área basal $\left(\mathrm{m}^{2}\right.$.ha-1 $)$ e densidade absoluta (ind.ha ${ }^{-1}$ ), que foram determinadas para o bosque e por espécie. Densidade relativa, dominância relativa, freqüência relativa e valor de importância foram estimados para cada espécie.

A relação entre altura e diâmetro foi examinada através de análise de regressão não linear, usando o programa Xact versão 7.21 d, e a similaridade entre as áreas de estudo foi determinada através da análise de agregação (cluster análise) com o uso do programa STATISTICA 6.0.

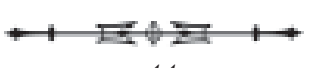




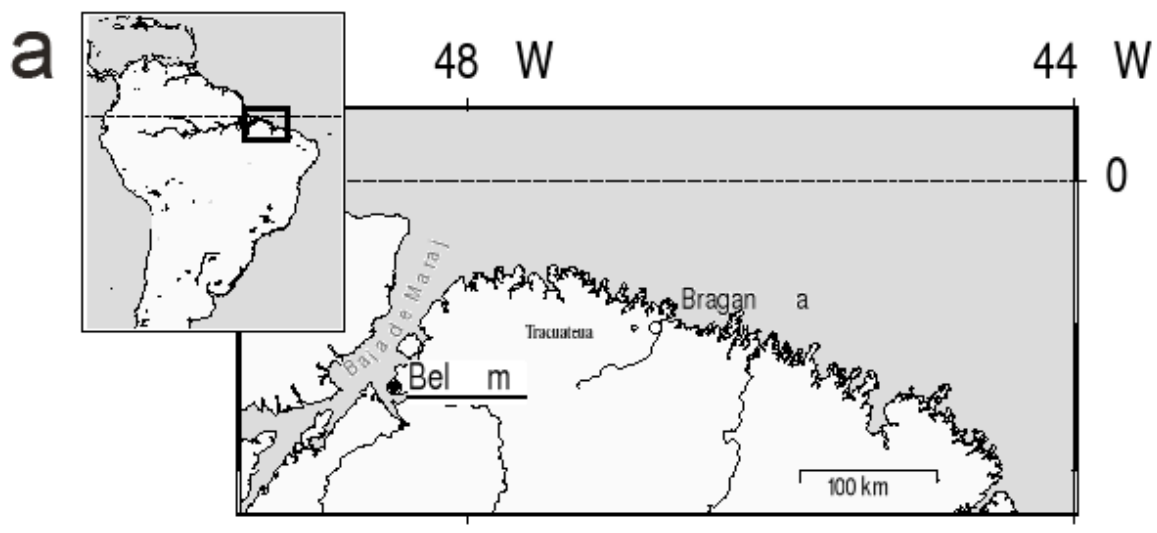

b

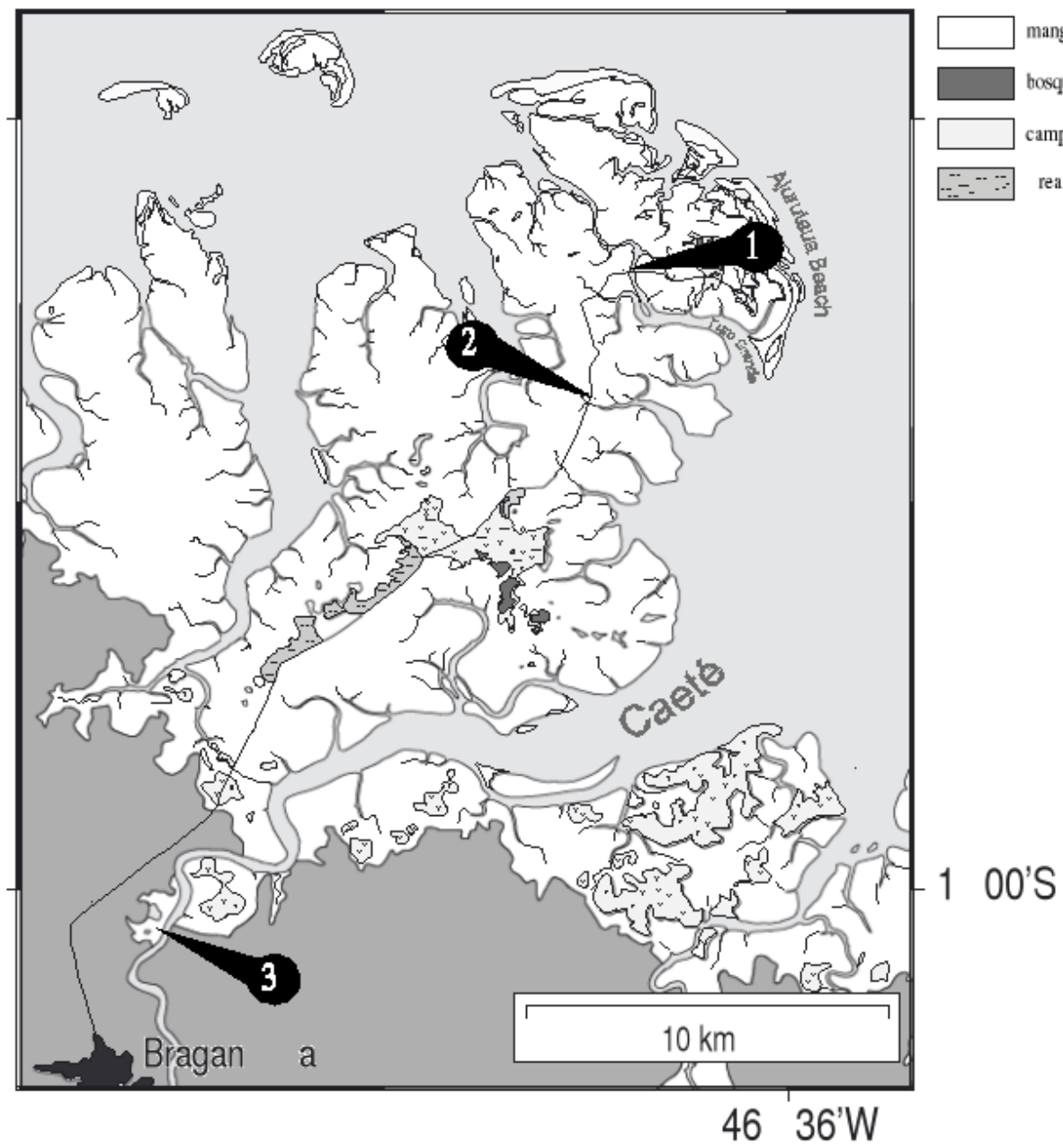

Figura 1. Localização das áreas de estudo a) costa do Pará e b) península de Bragança, Pará, Brasil. Área 1 - Furo Grande (FG); Área 2 - Furo do Meio (FdM); Área 3 - Acarajó (AC).

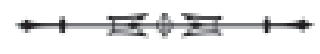




\section{RESULTADOS}

\section{Ocorrência de espécies}

Em todas as áreas estudadas ocorreram três espécies típicas de mangue: Rhizophora mangle $\mathrm{L}$. (Rhizophoraceae), Avicennia germinans (L.) Stearn 1958 (Avicenniaceae) e Laguncularia racemosa (L.) Gaertn. f. (Combretaceae). R. mangle foi a espécie mais abundante em todas as áreas (densidade relativa: 77,5\% AC, 63,8\% FdM; 85\% FG); $A$. germinans a segunda mais abundante (densidade relativa: 15\% AC, 23,8\% FdM; 10\% FG) ocorrendo principalmente em áreas mais altas; e, $L$. racemosa foi a espécie menos freqüente (densidade relativa: 7,5\% AC, 12,5\% FdM; 5\% FG), aparecendo principalmente em áreas de recolonização (clareiras e áreas degradadas). R. mangle apresentou Valores de Importância (VI) mais elevados em todas as áreas estudadas (Tabela 1).

\section{Parâmetros estruturais}

$\mathrm{Na}$ área $\mathrm{AC}, \mathrm{R}$. mangle apresentou maiores valores médios de diâmetro $(34 \mathrm{~cm} \pm 21)$ e altura (14,6 $\mathrm{m} \pm 5$ ), enquanto que, nas áreas FdM e FG, A. germinans apresentou os maiores valores para esses parâmetros $(43,2 \mathrm{~cm} \pm 21,7$ e 33,4 cm $\pm 16,1$ - diâmetro e 13,8 m \pm 3,1 e 14,8 m \pm 4,4 altura, respectivamente) (Tabela 1). Em todas as áreas estudadas $L$. racemosa apresentou os menores valores para os parâmetros estruturais.

FdM foi a área com maior valor de área basal (Tabela 1). Observando a distribuição das classes de diâmetro em cada área, percebe-se que na área $A C$ os indivíduos estão distribuídos em diferentes classes de forma mais equilibrada; em FG concentram-se entre 12,5 e 32,5 cm; enquanto na área FdM estão concentrados entre 12,5 e 42,5 cm DAP. É possível notar, também, a presença de indivíduos de elevado diâmetro tanto na área $A C$, que chega a $89,13 \mathrm{~cm}$, quanto na FdM, chegando a $95 \mathrm{~cm}$ de diâmetro. Considerando-se a altura, apesar da similaridade das médias, observa-se que na área FG há maior concentração de árvores entre 14 a 19 m, enquanto na FdM elas estão distribuídas entre 9 e $18 \mathrm{~m}$. Já na área $\mathrm{AC}$, a altura das árvores está distribuída entre 14 e 24 m (Figura 2). As análises de regressão entre altura e diâmetro mostram que o crescimento em altura é limitado, enquanto o crescimento em diâmetro é contínuo (Figura 3). Na área AC as árvores crescem concomitantemente em altura e diâmetro até o ponto

Tabela 1. Dados estruturais dos bosques estudados. Local: AC-Acarajó; FdM-Furo do Meio; FG-Furo Grande; Sp-espécie; LgLaguncularia racemosa; Av-Avicennia germinans; Rh-Rhizophora mangle; Dens. Abs-densidade absoluta; Dens. Rel-denisdade relativa; Dom rel-dominância relativa; Freq. Rel-dominância relativa; VI-valor de importância.

\begin{tabular}{|c|c|c|c|c|c|c|c|}
\hline $\begin{array}{l}\text { Local/ } \\
\text { espécie }\end{array}$ & $\begin{array}{l}\text { Dens abs } \\
\text { ind/ha }\end{array}$ & $\begin{array}{c}\text { A. Basal } \\
\mathrm{m}^{2} / \mathrm{ha}\end{array}$ & Freq abs & Dens rel & Dom rel & Freq rel & VI \\
\hline$\overline{A C}$ & 101,6 & 107 & - & - & - & - & - \\
\hline $\operatorname{Lg}$ & 7,6 & 0,3 & 30 & 7,5 & 0,3 & 0,18 & 7,98 \\
\hline Av & 15,1 & 16 & 40 & 15 & 15,13 & 0,24 & 30,37 \\
\hline $\mathrm{Rh}$ & 78,3 & 91 & 95 & 77,5 & 82,58 & 0,58 & 162,66 \\
\hline FdM & 146 & 111 & - & - & - & - & - \\
\hline $\operatorname{Lg}$ & 18 & 3 & 35 & 12,5 & 3,12 & 0,15 & 15,77 \\
\hline$A v$ & 34,7 & 64 & 100 & 23,75 & 57,47 & 0,43 & 81,65 \\
\hline $\mathrm{Rh}$ & 93,1 & 44 & 100 & 63,75 & 39,41 & 0,43 & 103,58 \\
\hline FG & 79,4 & 47 & - & - & - & - & - \\
\hline $\operatorname{Lg}$ & 4 & 1 & 15 & 5 & 1,78 & 0,1 & 6,88 \\
\hline$A v$ & 7,9 & 8 & 35 & 10 & 17,82 & 0,23 & 28,05 \\
\hline $\mathrm{Rh}$ & 67,5 & 38 & 100 & 85 & 80,4 & 0,67 & 166,06 \\
\hline
\end{tabular}

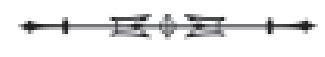



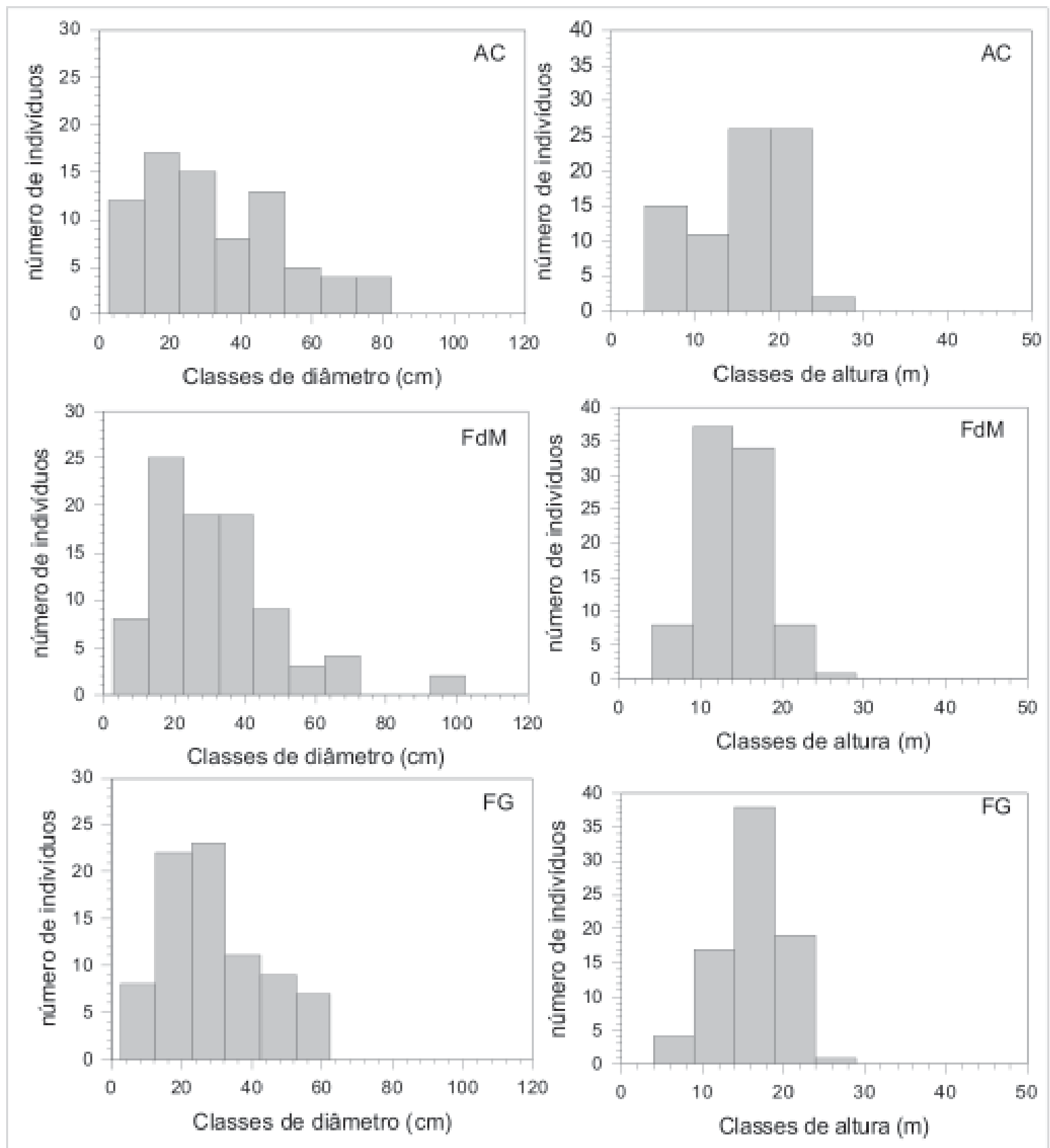

Figura 2. Distribuição das classes de altura $(\mathrm{m})$ e diâmetro $(\mathrm{cm})$ das árvores estudadas (AC, FdM e FG) na península de Bragança, Pará, Brasil.

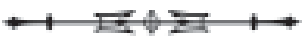



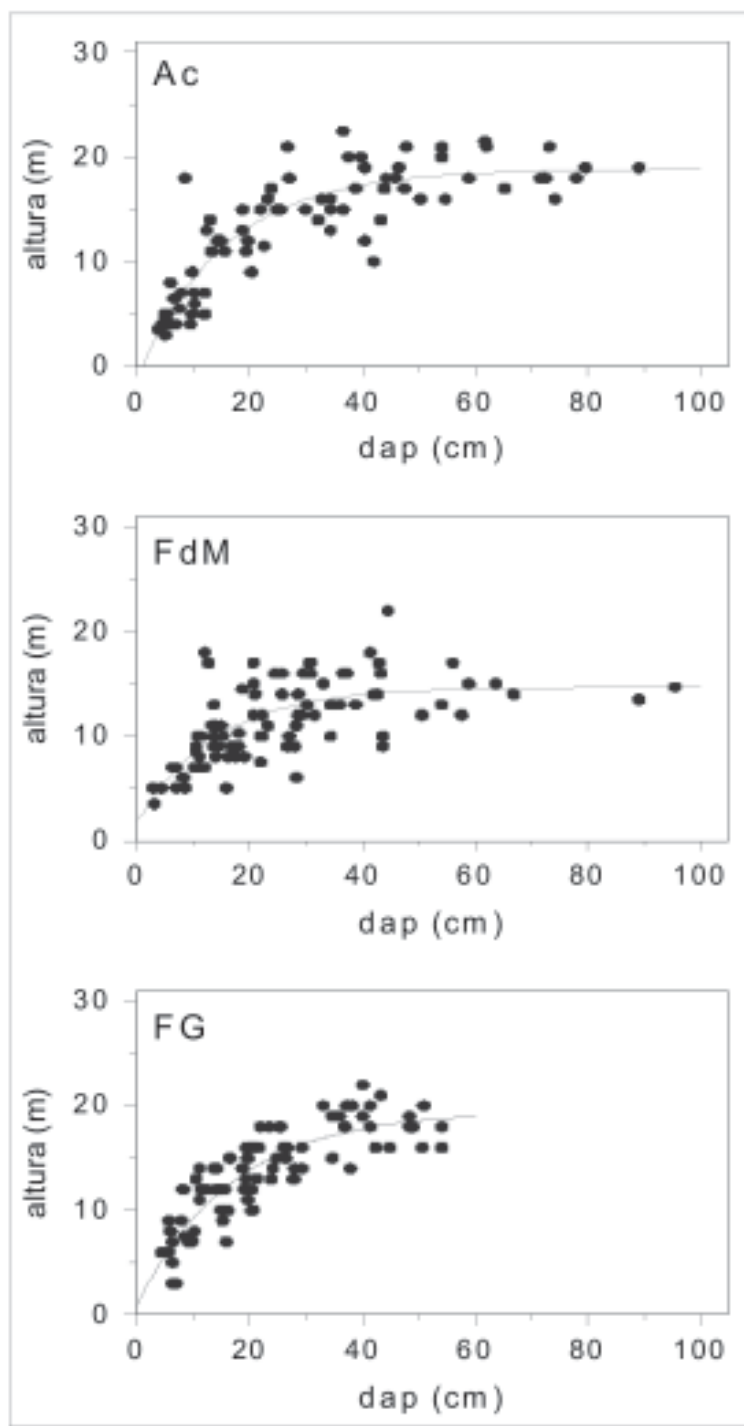

Figura 3. Regressão não linear entre altura e diâmetro das árvores das três áreas de estudo na pensínsula de Bragança.

em que atingem o tamanho de $37 \mathrm{~cm}$ de diâmtero $(r=0,88)$. Na área FG, altura e diâmetro crescem simultaneamente até atingirem $38 \mathrm{~cm}$ de diâmetro $(r=0,86)$, e na FdM alcaçaram $31 \mathrm{~cm}$. A densidade geral das áreas foi 101 ind.ha-1 $^{-1}(A C), 146$ ind.ha-1 $^{-1}$ (FdM) e 79,4 ind.ha-1 (FG). Em uma análise da densidade de cada ponto amostrado para cada uma das três áreas, nota-se que os padrões de distribuição das árvores difere entre as áreas (Figura 4). Em FdM, por exemplo, nos primeiros oito pontos a densidade é mais elevada e irregular, enquanto no AC e FG a distribuição das árvores apresenta-se bastante homogênea ao longo da transecção.

Quando as características estruturais (composição de espécies, diâmetro, altura, área basal e inundação) dos bosques são examinadas, através da análise de agrupamento, há dois grupos: um formado pela área FdM e outro pelas áreas $A C$ e FG (Figura 5).

\section{DISCUSSÃO}

As espécies típicas de mangue encontradas nas áreas estudadas são as mesmas descritas por Menezes, Berger e Worbes (2003), Seixas (2003) e Abreu (2004) para diferentes áreas da península bragantina. Estas mesmas espécies também foram encontradas por Mello et al. (1995), Almeida (1996), Bastos e Lobato (1996), Costa Neto e Senna (2000) para outras regiões da costa paraense. Amaral et al. (2001) e Santos et al. (2003) apontam a ocorrência de $A$. schaueriana na ilha de Canelas e praia de Ajuruteua, respectivamente, ainda que esta espécie apresente apenas indivíduos de pequeno porte, não tendo sido encontrada nas áreas do presente estudo. Almeida (1996) sugere que no nordeste do Pará, em manguezais sob influência marinha e altos teores de salinidade, as espécies arbóreas encontradas são $R$. mangle, A. germinans e L. racemosa.

No presente trabalho é possível constatar, pelos altos valores de $\mathrm{VI}$ (Tabela 1), que os manguezais estudados são bosques de $R$. mangle, pois esta espécie apresentou os maiores valores deste índice em todas as áreas de estudo, tal como encontrado por Seixas (2003), Menezes, Berger e Worbes (2003) e Abreu (2004). No presente estudo, R. mangle alcançou maiores valores de diâmetro, altura e área basal na área de manguezal salobro (AC), enquanto A. germinans apresenta maiores valores nos bosques das áreas FdM e FG. Esta constatação provavelmente indica que $R$. mangle desenvolve-se melhor em condições de baixa salinidade. Bosques dominados

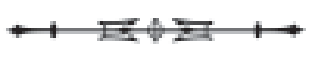




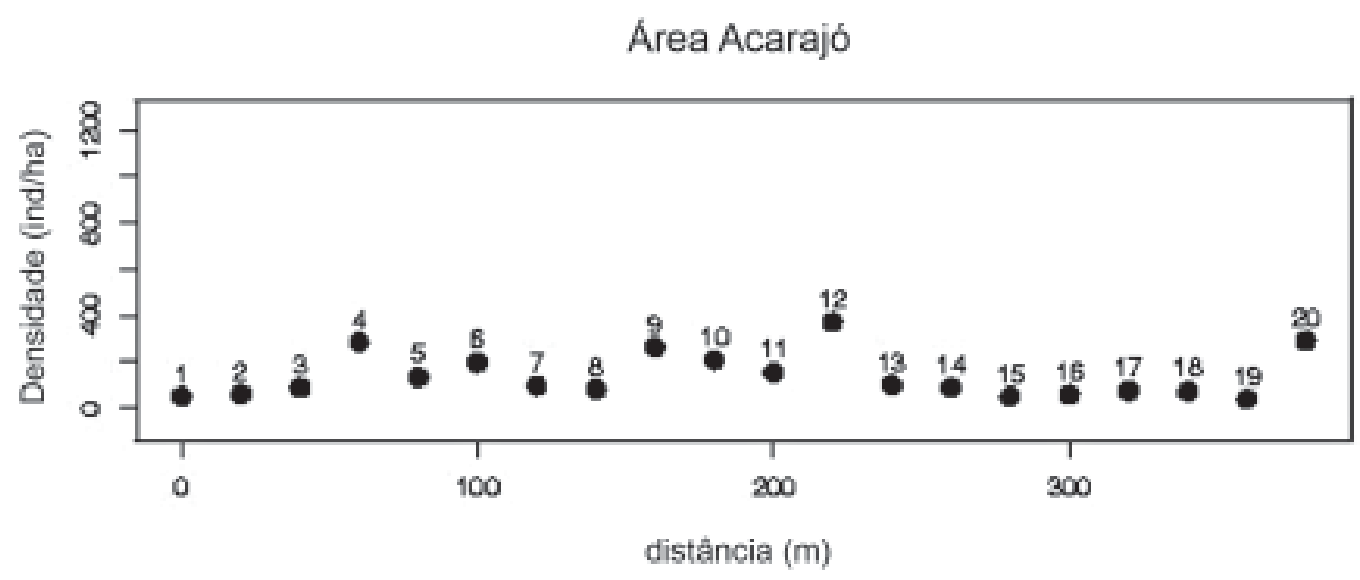

Área Furo Grande

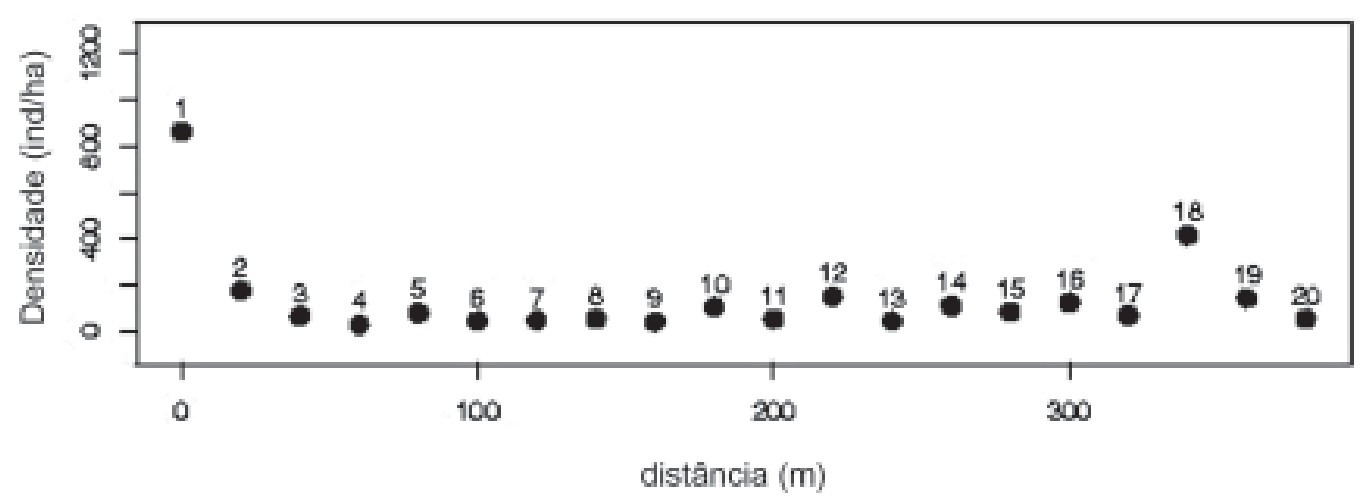

Área Furo do Melo

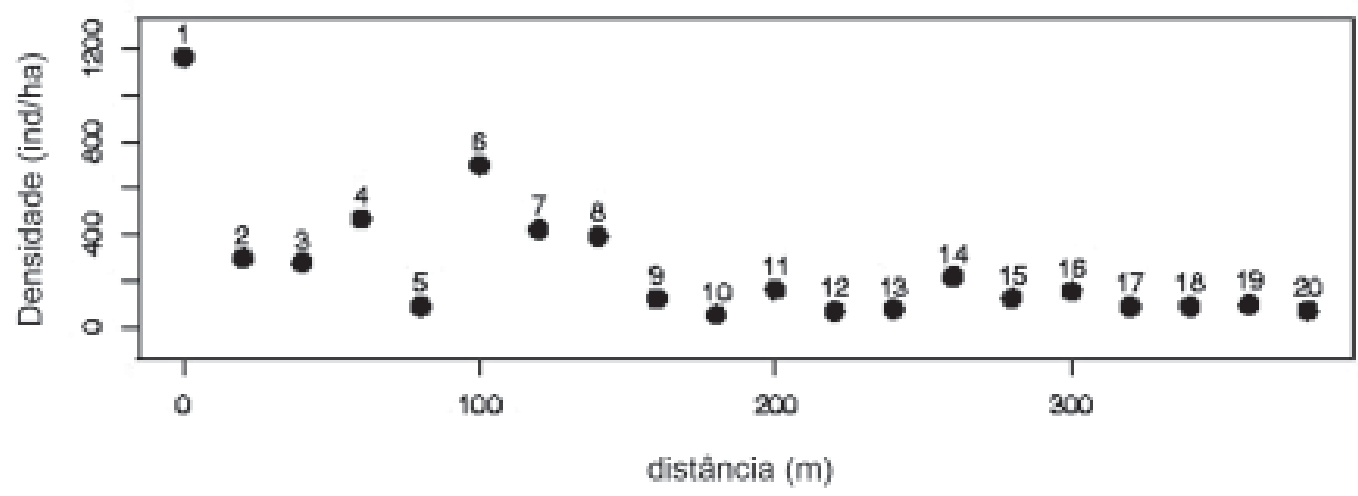

Figura 4. Densidade (ind.ha-1 ${ }^{-1}$ ) de cada quadrante das transecções demarcadas nas áreas de estudo.

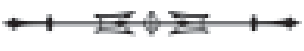




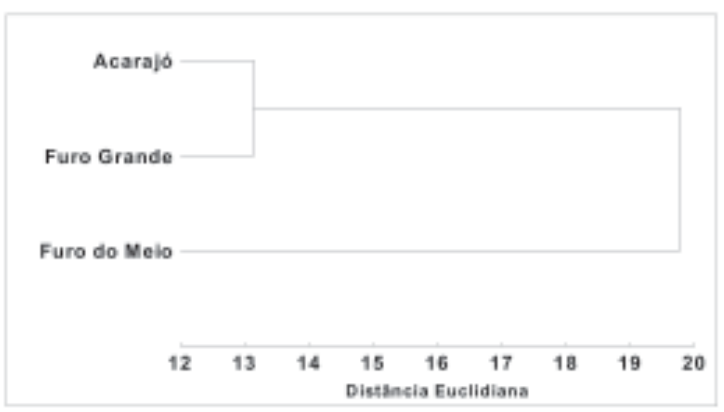

Figura 5. Dendrograma de agregação (características estruturais, distribuição de espécies e freqüência de inundação) das áreas de mangue estudadas na península de Bragança.

por R. mangle também são descritos para outras áreas da costa paraense, como Marapanim (COSTANETO; SENNA, 2000) e Crispim (BASTOS; LOBATO, 1996). L. racemosa sempre aparece como parte menor do bosque, com presença em bordas de canais e clareiras. Esta espécie é a pioneira na recolonização de áreas de clareiras nos bosques de mangue da área AC (ADAMS; BERGER, 2002) e coloniza áreas de manguezais degradados com deficiência de inundação na península de Bragança (MENEZES; COMPTON, 2003; CARVALHO, 2000). Esta espécie também é citada colonizando bancos de lama na Guiana Francesa (FROMARD et al., 1998) e no Maranhão, onde também é descrita colonizando áreas degradadas (MOCHEL et al., 2001). Excessão parecem ser os mangues da itha de Maracá, onde esta espécie forma bosques monoespecíficos de grande porte (FERNANDES, 1997). A. germinans aparece como a segunda espécie em ocorrência em todos os mangues estudados na península, porém, na FdM há um alto VI, valor bem próximo do VI registrado para R. mangle nesta área. Segundo Lugo e Snedaker (1974), a distribuição das espécies de mangue está relacionada à inundação. $R$. mangle desenvolve-se melhor em áreas de frequente inundação, enquanto A. germinans é capaz de sobreviver em áreas pouco inundadas (TOMLINSON, 1986).

Os dados de diâmetro e altura mostram que os bosques estudados apresentam grande porte se comparados aos mangues estudados por Seixas (2003) e Abreu (2004). Os altos valores de área basal observados no FdM são decorrentes dos grandes diâmetros das árvores de $A$. germinans. A distribuição dos valores de diâmetro evidenciam a diferença estrutural dos bosques. Em AC há presença de indivíduos de pequeno porte, assim como de médio e grande porte, o que pode ser indício de que na área há um processo constante de renovação. $\mathrm{Na}$ área FdM observa-se a existência de vários indivíduos de porte médio, o que pode ser indício de que em um determinado período houve uma grande renovação do bosque, embora a existência de grandes árvores mostrem tratar-se de um bosque antigo. $\mathrm{Na}$ área FG os bosques parecem ainda estar em fase de crescimento, pois há muitas árvores de porte médio.

Assumindo o ponto em que árvores cessam o crescimento em altura como o momento em que estas atingem a idade madura, chamar-se-á este momento de 'ponto de maturação'. Partindo deste princípio e considerando que o diâmetro médio das árvores das áreas FdM e AC (Tabela 1) está próximo ao ponto de maturação, assume-se que este bosques são maduros, pois estão apenas crescendo em diâmetro. Por outro lado, o diâmtero médio das árvores do FG está abaixo do ponto de maturação (Tabela 1), por isso este bosque é considerado jovem.

A densidade de árvores dos bosques deste estudo parece baixa quando comparada aos resultados de Menezes, Berger e Worbes (2003). Porém, quando a distribuição das árvores nas classes de diâmetro e altura são consideradas, nota-se que os bosques apresentam árvores de grande porte, o que pode ser refletido na baixa densidade.

A ocorrência de dois grupos de bosques, como mostrado pelo dendrograma, é um reflexo das características estruturais e de inundação das áreas estudadas. A semelhança entre as áreas AC e FG está na composição de espécies, estrutura do bosque e na inundação mais freqüente. A área FdM diferencia-se pelos altos valores de dominância relativa de

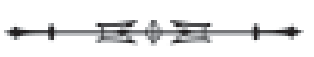


A. germinans, diferenciando-se das outras áreas, onde $R$. mangle foi a espécie com maiores valores nos parâmetros estruturais, além de apresentar valores inferiores na freqüência de inundação.

\section{CONCLUSÃO}

Pode-se dizer que a península de Bragança apresenta bosques de mangue de grande porte, dominada por R. mangle. $\bigcirc$ crescimento em altura é limitado, enquanto o crescimento em diâmetro é contínuo. AC mostrou tratar-se de um bosque maduro com renovação constante. FdM trata-se de um bosque com um pico de colonização em um determinado período do passado. Na área FG dominam árvores de porte médio e a relação ente altura e diâmetro sugere tratar-se de um bosque jovem. Finalmente, freqüência de inundação parece ser um fator importante na diferenciação estrutural entre os bosques, pois as áreas com inundação mais freqüente agruparam-se juntas na análise de agregação.

\section{AGRADECIMENTOS}

O primeiro autor agradece ao PIBIC/UFPA pela concessão da bolsa de iniciação científica. Este trabalho foi financiado pelo CNPq (Brasil) e BMBF (Alemanha), através do Projeto Mangrove Dynamics and Management (MADAM), um projeto de cooperação bilateral Brasil-Alemanha.

\section{REFERÊNCIAS}

ABREU, M. M. O. 2004. Caracterização estrutural e composição florística em um bosque de terra firme e um manguezal da peninsula de Ajuruteua, Bragança, Pará, Brasil. Monografia (Trabalho de Conclusão de Curso em Licenciatura em Biologia) - Universidade Federal do Pará, Campus de Bragança. ADAMS, M.; BERGER, U. 2002. Regeneração e sucessão em áreas de manguezal após cultivo de arroz Bragança-Pará. In: WOKSHOP ECOLAB, 6., Resumos expandidos... [S.l.s.n.n.] 1 CD ROM.

ALMEIDA, S. 1996. Estrutura e florística em áreas de manguezais paraenses: evidências da influência do estuário amazônico. Bol. Mus. Para. Emílio Goeldi, sér. Cienc. da Terra, v. 8, p. 93-100.
AMARAL, D. D. et al. 2001. A vegetação da Iha de Canela, município de Bragança - Pará - Brasil. Bol. Mus. Para. Emilio Goeldi. sér. Bot. v. 17, n. 2, p. 389-402.

BASTOS, M. N.; LOBATO, L. C. 1996. Estudos fitossociológicos em áreas de bosque de mangue na praia do Crispim e Ilha de Algodoal. Bol. Mus. Para. Emílio Goeldi, sér. Cienc. da Terra, v. 8, p. 157-167.

CARVALHO, E. A. 2000. Impactos ambientais na zona costeira: o caso da estrada Bragança-Ajuruteua, estado do Pará. Dissertação (Mestrado em pós-graduação em Ciencia Ambiental) - Universidade de São Paulo, PROCAM.

COSTA NETO, S. V.; SENNA, C. 2000. Estrutura e diversidade de bosques de manguezal da baía de Marapanim. [S.1.:s.n.]. Full Papers. 1 CD ROM. Mangrove 2000 - Conference.

FERNANDES, M. E. B. 1997. The ecology and productivity of mangroves in the Amazon region. Tese (Doutorado) - University of York, England.

FROMARD, F. et al. 1998. Structure, above-ground biomass and dynamics of mangrove systems: new data from French Guiana. Oecologia, v. 115, p. 39-53.

KRAUSE, G.; SCHORIES, D.; DIELE, K. 2001. Spatial patterns of mangrove ecosystems: the bragantinian mangroves of northern Brazil (Bragança, Pará). Ecotropica, v. 7, p. 93-107.

LUGO, A. E.; SNEDACKER, S. C. 1974. The ecology of mangroves. Annual review of ecology and systematics, v. 5, p. 39-64.

MELLO, C. F. et al. 1995. As angioespermas de alguns manguezais paraenses. In: WORKSHOP ECOLAB, 3. Livro de Resumos expandidos... [S.l.:s.n.].

MENEZES, M. P. M.; COMPTON, A. T. 2003. An analysis of the vegetation related to the inundation level of degraded mangrove forest: Bragança peninsula, NE-Brazil. [S.l.:s.n.]. Livro de Resumos. Mangrove 2003 - Conference.

MENEZES, M. P. M.; BERGER, U.; WORBES, M. 2003. Annual growth rings and long-term growth patterns of mangrove trees from the Bragança peninsula, NE Brazil. Wetlands, v. 11, p. 233-242.

MOCHEL, F. R. et al. 2001. Degradação dos manguezais da Itha de São Luis (MA): processos naturais e impactos antrópicos. In: ECOSSISTEMAS costeiros: impactos e gestão ambiental. Belém: MPEG, FUNTEC.

PROST, M. T.; RABELO, B. V. 1996. Variabilidade fito-espacial de manguezais litorâneos e dinâmica costeira: exemplos da Guiana Francesa, Amapá e Pará. Bol. Mus. Para. Emilio Goeldi, sér. Cienc. da Terra, v. 8, p. 101-121.

SANTOS, C. C. L. et al. 2003. Fenologia de Avicennia germinans e Avicennia schaueriana na praia de Ajuruteua, nordeste do Pará. In: CONGRESSO BRASILEIRO DE BOTÂNICA, 55. Anais... [S.l.s.s.n.]. SCHAEFFER-NOVELU, Y.; CINTRÓN, G. 1986. Guia para estudos de áreas de manguezais (estrutura, função e flora). [S.l.]: Caribean Ecological Research. 156 p.

SEIXAS, J. A. S. 2003. Estudo comparativo da estrutura da vegetação dos bosques de mangue do Furo Grande, Bragança, Pará. Monografia (Trabalho de Conclusão de Curso Licenciatura em Biologia) - Universidade Federal do Pará, Campus de Bragança.

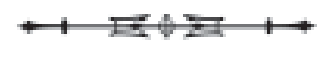


SOUZA-FLLHO, P. W. 2005. Costa de manguezais de macro-mari da Amazônia: cenários morfológicos, mapeamento e quantificação de áreas usando dados e sensores remotos. Revista Brasileira de Geofísica. v. 23, n. 4, p. 427-435.

SOUZA-FILHO, P. W. 1995. A planície costeira bragantina (NE do Pará): influência das variações do nível do mar na morfoestratigrafia costeira durante $\bigcirc$ Holoceno. Tese (Mestrado em geologia e geoquímica) - Universidade Federal do Pará. TOMLINSON, P. B. 1986. The botany of mangroves. Cambridge: Cambridge University Press. 418 p. 